\title{
Optimising Electron Holography in the Presence of Partial Coherence and Instrument Instabilities with Conventional and Direct Detection Cameras
}

\author{
Shery L. Y. Chang ${ }^{1}$, Christian Dwyer ${ }^{1}$, Juri Barthel ${ }^{1}$, Chris B. Boothroyd ${ }^{1}$ and Rafal E. Dunin- \\ Borkowski $^{1}$ \\ 1. Ernst Ruska-Centre for Microscopy and Spectroscopy with Electrons, and Peter Gruenberg Institute, \\ Forschungszentrum Juelich, Juelich, Germany
}

Off-axis electron holography provides a direct means of retrieving the phase of the wavefield in a transmission electron microscope, enabling the measurement of electric and magnetic fields at length scales from microns to nanometers. To maximise the precision of the technique, it is important to acquire holograms using experimental conditions that optimise the phase resolution for a given spatial resolution. These conditions are determined by a number of competing parameters, especially the spatial coherence and the instrument stability.

Here, we describe a simple, yet accurate, model for predicting the dose rate and exposure time that give the best phase resolution in a single hologram. Experimental studies were undertaken to verify the models of spatial coherence and instrumental instabilities that are required for the optimisation. The model is applicable to holography in both standard mode and Lorentz mode, and it is relatively simple to apply.

In light of the considerable body of literature describing the factors governing the phase resolution in electron holography [1-3], we emphasize that we kept the models of spatial coherence and instrument instabilities as simple as possible. In addition, we have evaluated the performance of a state-of-the-art direct detection camera (DDC) for electron holography, and compare its performance in various operational modes with a conventional integrating charge coupled device (CCD) camera.

We found that the commonly-used Gaussian model [2] is not suitable to describe the spatial coherence, and instead a bivariate Cauchy distribution convoluted with a Gaussian distribution is better suited. The fringe movement due to instabilities is well-modelled by the Langevin theory of Brownian motion, which improves upon previous models [4-5] since it is applicable to the practical range of exposure times used in experiments. These models can be determined with two data sets with a fixed biprism voltage and magnification: an intensity series (Fig. 1) to determine the spatial coherence and a time series (Fig. 2) to determine the time-dependent visibility. The optimum dose rate and exposure time (not considering specimen instabilities) for other combinations of biprism voltages and magnifications can then be predicted.

The comparison of the phase errors at the optimum conditions (at $300 \mathrm{kV}$ ) between a conventional CCD camera (UltraScan, Gatan) and a DDC camera (K2, Gatan) for a given exposure time (1sec) shows a two-fold reduction in the phase error. This improvement is largely due to the improvement of DQE and MTF offered by the DDC. This suggests that the use of DDC can have significant advantages in the application of electron holography. 


\section{References:}

[1] H Lichte et al, Optik 77 (1987) p. 135.

[2] H Lichte, Ultramicroscopy 108 (2008) p. 256.

[3] F. Roeder et al, Ultramicroscopy 144 (2014) p. 32.

[4] E. Voelkl, Ultramicroscopy 110 (2010) p. 199

[5] R. A. McLeod et al, Ultramicroscopy 141 (2014) p. 38.
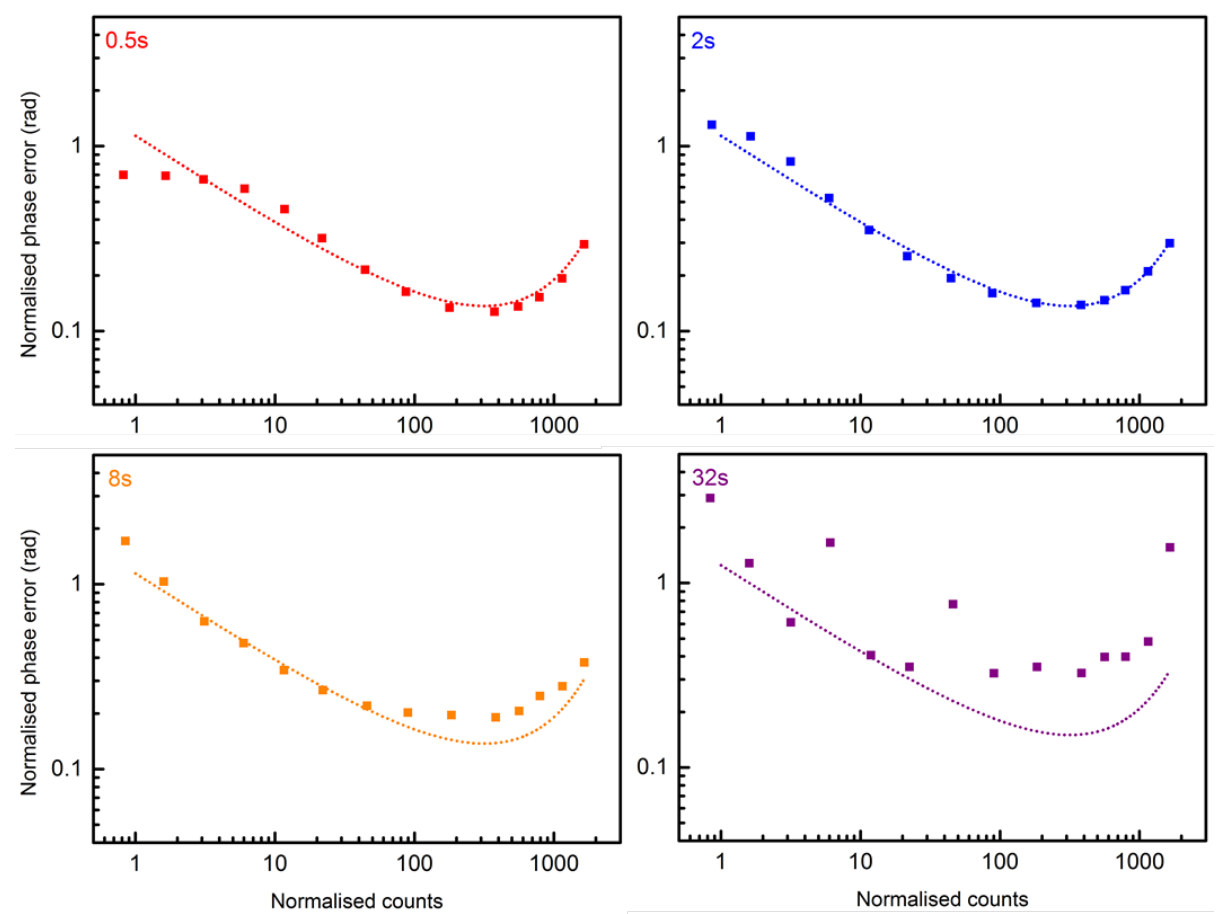

Figure 1. The normalized phase error as a function of normalized signal, plotted for selected exposure times (indicated in each graph). Also shown are predictions of phase errors (dotted lines) based on the fitting of the theoretical models.
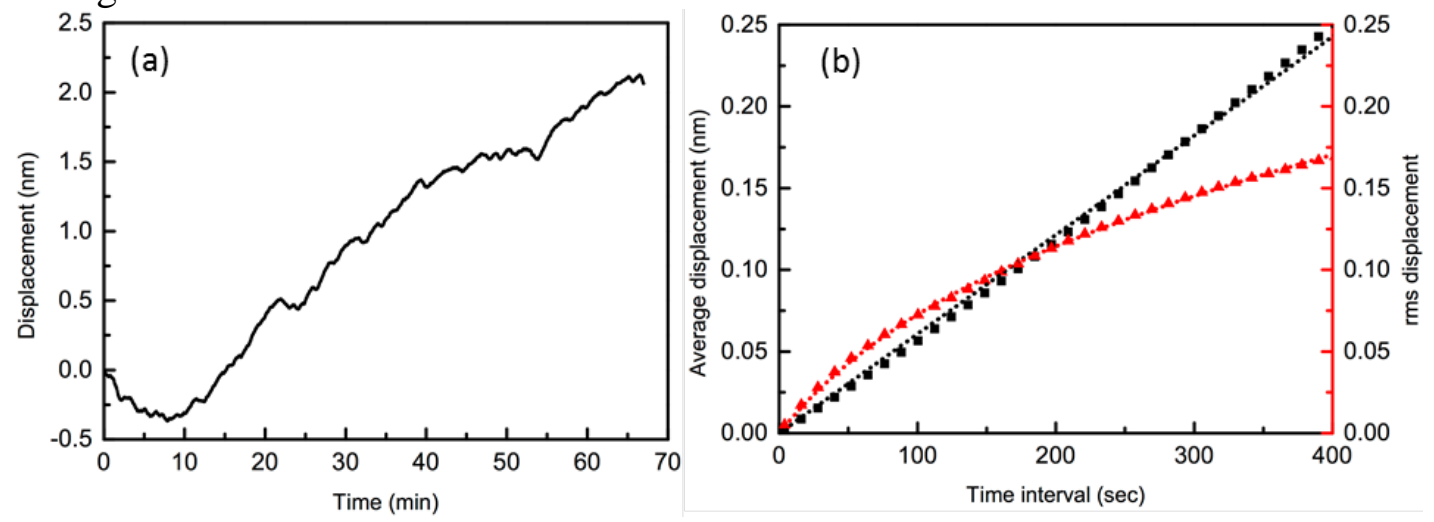

Figure 2. (a) Fringe displacement as a function of time, measured using 1000 holograms over a timespan of 67 minutes. (b) The average (black squares) and rms (red triangles) displacement for time intervals up to 400 seconds. The theoretical models are overlaid (dotted lines). 